\title{
Factores asociados a desnutrición en pacientes hospitalizados en el servicio de cirugía de emergencia de un hospital del seguro social peruano Malnutrition-associated factors in patients hospitalized in an emergency surgical ward in a Peruvian social security hospital
}

Correspondencia Cristian Díaz Vélez cristian.diaz@essalud.gob.pe

\section{Recibido: 13/03/2020}

Arbitrado por pares

Aprobado: 20/08/2020

Citar como: Zeña-Huancas PA, Pajuelo-García D, Díaz-Vélez C Factores asociados a desnutrición en pacientes hospitalizados en el servicio de cirugía de emergencia de un hospital del seguro social peruano. Acta Med Peru. 2020;37(3):278-84. doi: https://doi org/10.35663/amp.2020.372.1849

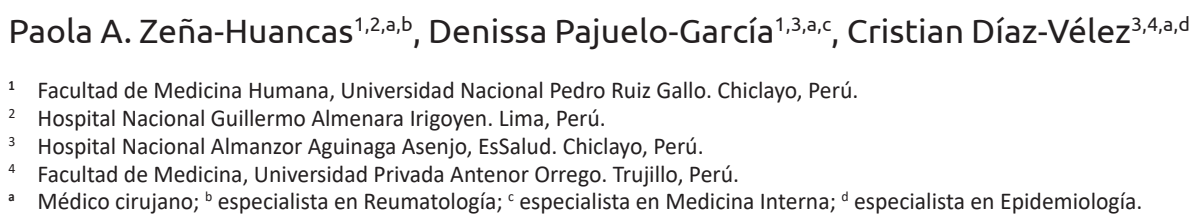

\section{RESUMEN}

Objetivo: identificar los factores asociados a desnutrición en pacientes hospitalizados en el Servicio de Cirugía de Emergencia del Hospital Nacional Guillermo Almenara Irigoyen. Materiales y métodos: estudio analítico donde se realizó un tamizaje inicial mediante el Nutritional Risk Screening 2002, posteriormente una evaluación nutricional al ingreso y egreso mediante la Valoración global subjetiva en 206 pacientes adultos y adultos mayores. Se analizaron variables clínicas mediante regresión logística y un valor de $p<0,05$ fue considerado como significativo. Resultados: el $16 \%$ de pacientes agravó su estado nutricional durante la hospitalización. Los factores asociados fueron alguna comorbilidad (odds ratio [OR] 2,32; intervalo de confianza [IC]95\%:1,07-5,01); neoplasias (OR: 2,83; IC95\%:1,21-6,63), enfermedades del sistema nervioso (OR: 5,66; IC95\%: 1,24-25,82); ausencia del registro de peso y talla (OR: 4,18; IC95\%: 1,29-13,41); cirugía abdominal (OR: 6,67; IC95\%: 3,08-14,89); apendicitis aguda disminuía 85\%; colecistitis aguda disminuía 91\%; reintervención quirúrgica (OR: 13,91; IC95\%: 4,82-40,06); consumo regular dieta indicada (OR: 8,78; IC 95\%: 3,01-25,63); no consumir dieta indicada (OR: 39,97; IC95\%: 11,39-140,23); uso de soporte nutricional disminuía la exposición en un $83 \%$. La desnutrición incrementaba 6 veces el riesgo de mortalidad. El número de días de ayuno, (OR: 1,73; IC95\%: $1,31-2,27)$. La mortalidad fue $8,7 \%$. Conclusiones: existen una serie de factores asociados a la desnutrición durante la hospitalización, por lo que es necesario realizar un tamizaje para la detección temprana de desnutrición al ingreso de los pacientes que serán hospitalizados y posteriormente una evaluación nutricional en aquellos que estén en riesgo.

Palabras clave: Desnutrición; Hospitalización; Cirugía (fuente: DeCS BIREME). 


\section{ABSTRACT}

Objective: to identify malnutrition-associated factors in patients hospitalized in the emergency surgical ward in Guillermo Almenara-Irigoyen National Hospital. Materials and methods: this is an analytical study in which an initial nutritional evaluation was performed using the 2002 Nutritional Risk Screening. Afterwards, a nutritional assessment was performed on admission and at discharge using a subjective overall assessment in 206 adult and elderly subjects. Clinical variables were analyzed using logistic regression and $p<0.05$ was considered as significant. Results: sixteen per cent of all patients worsened their nutritional status during hospitalization. Associated factors were some comorbidity (odds ratio [OR] 2.32; 95\% confidence interval [CI]: 1.07-5.01); neoplasms (OR: 2.83; 95\% Cl: 1.21-6.63), nervous system diseases (OR: 5.66; 95\% $\mathrm{Cl}: 1.24-25.82$ ); absence of weight and height recording (OR: 4.18; $95 \% \mathrm{Cl}: 1.29-13.41)$; abdominal surgery (OR: $6.67 ; 95 \% \mathrm{Cl}$ : 3.08-14.89); acute appendicitis decreased 85\%; acute cholecystitis decreased 91\%; surgical reintervention (OR: 13.91; 95\% $\mathrm{Cl}: 4.82-40.06)$; regular consumption of the indicated diet (OR: $8.78 ; 95 \% \mathrm{Cl}: 3.01-25.63)$; not consuming the indicated diet (OR: $39.97 ; 95 \% \mathrm{Cl}$ : $11.39-140.23$ ); use of nutritional support decreased exposure by $83 \%$. Malnutrition increased the risk of mortality 6 times. The number of days of fasting, (OR: $1.73 ; 95 \% \mathrm{Cl}: 1.31-2.27)$. Mortality was $8.7 \%$. Conclusions: there are some factors associated to malnutrition during hospitalization, so it is necessary to perform screening for early detection of malnutrition when patients are admitted, and also, nutritional assessments of those found to be at risk are necessary..

Keywords: Malnutrition; Hospitalization; Surgery (source: MeSH NLM).

\section{INTRODUCCIÓN}

La desnutrición hospitalaria con frecuencia no se diagnostica; sin embargo, estudios internacionales, han estimado la prevalencia de desnutrición entre el 20 y $50 \%$ en los pacientes adultos hospitalizados ${ }^{[1]}$. En el Perú, Vinatea et al. reportan que la desnutrición en el Hospital Nacional Guillermo Almenara Irigoyen (hospital de alta complejidad en Lima, Perú) es de $42 \%{ }^{[2]}$; y en pacientes intervenidos por cirugía gastrointestinal, la prevalencia fue similar ${ }^{[3]}$. Entre los factores asociados se encuentra la edad (sobre todo mayores de 60 años) ${ }^{[4]}$, comorbilidades y cirugía ${ }^{[5]}$.

La evaluación nutricional no forma parte de la práctica rutinaria en la mayoría de los hospitales generando la ausencia o retraso del registro del peso y talla en las historias clínicas ${ }^{[6]}$. Cerca de un $30 \%$ del aporte de la dieta indicada no es ingerido por anorexia, vómitos, náuseas y síntomas depresivos ${ }^{[5]}$. Los pacientes desnutridos tienen, en promedio, estancias hospitalarias más prolongadas $\left(17,2\right.$ días) comparado con pacientes no desnutridos $(9,1 \text { días })^{[7]}$.

Se recomienda que en las primeras 24 a 48 horas de ingreso, se debe realizar un tamizaje para la detección temprana de desnutrición ${ }^{[8,9]}$. La Federación Latinoamericana de Terapia Nutricional, Nutrición Clínica y Metabolismo (FELANPE); recomienda el empleo del Nutritional Risk Screening 2002 (NRS 2002) para pacientes hospitalizados, que ha demostrado una elevada validez predictiva ${ }^{[10]}$; y sensibilidad mayor al $80 \%$ en pacientes de distintas patologías y edades, así como en mayores de 60 años ${ }^{[11]}$. Después de la etapa de clasificación inicial, se requiere una evaluación nutricional, encontrándose que, la valoración global subjetiva (VGS) es el instrumento de evaluación más adecuado en los diferentes entornos, siendo considerado como el estándar para evaluación nutricional hospitalaria ${ }^{[10,11]}$.

Hoy, se conoce que la desnutrición incrementa las complicaciones, la morbilidad y mortalidad del paciente hospitalizado, pero además podría existir un escaso conocimiento e interés del personal de salud sobre el estado nutricional de los pacientes. Para identificar en la práctica clínica a los pacientes desnutridos, se debería realizar una detección nutricional precoz, que permitiría no sólo diagnosticar diferentes grados de desnutrición, sino también distinguir entre pacientes que necesitan terapia nutricional y aquellos que no la precisan ${ }^{[12]}$.

El presente estudio tiene como objetivo identificar los factores asociados a desnutrición en pacientes hospitalizados en el Servicio de Cirugía de Emergencia del Hospital Nacional Guillermo Almenara Irigoyen.

\section{MATERIALES Y MÉTODOS}

\section{Diseño y estudio}

Se realizó un estudio prospectivo con muestreo por conveniencia de los pacientes del Servicio de Cirugía de Emergencia del Hospital Nacional Guillermo Almenara Irigoyen.

\section{Procedimiento para recolección de datos}

Se realizaron entrevistas junto con la recopilación de los datos registrados en las historias clínicas de los pacientes y cuaderno de ingresos del servicio de nutrición, la recopilación estuvo a cargo de uno de los autores, el cual, tuvo una capacitación en el uso de los instrumentos NRS 2002 y VGS en la Unidad de Soporte Nutricional del Hospital Nacional Guillermo Almenara Irigoyen, obteniéndose una concordancia adecuada.

Dentro de las 24 horas del ingreso, se realizó el tamizaje nutricional con el NRS 2002 y VGS. El NRS 2002 clasifica a los pacientes con una puntuación mayor o igual a tres para aquel paciente que está en riesgo nutricional y se debe iniciar un plan 
Tabla 1. Evolución del estado nutricional de los pacientes durante la hospitalización en el servicio de cirugía de emergencia de un hospital del seguro social peruano

\begin{tabular}{lccc}
\multirow{2}{*}{\multicolumn{1}{c}{ Ingreso }} & \multicolumn{2}{c}{ Egreso } \\
\cline { 2 - 4 } & Bien nutrido (A)* & Desnutrición moderada (B)* & Desnutrición severa (C)* \\
\hline Bien nutrido (A) & $123(59,7 \%)$ & $12(5,8 \%)$ & $0(0 \%)$ \\
Desnutrición moderada (B) & $8(3,9 \%)$ & $33(16,1 \%)$ & $21(10,2 \%)$ \\
Desnutrición severa (C) & $0(0 \%)$ & $4(1,9 \%)$ & $5(2,4 \%)$ \\
\hline
\end{tabular}

* Según la Valoración Global Subjetiva

nutricional y con puntuación menor de tres a aquellos pacientes que necesitan una monitorización semanal. Por otro lado, la VGS clasifica a los pacientes en nutrido (A), desnutrido moderado (B) y desnutrido grave $(C)^{[13,14]}$.

\section{Criterios de inclusión y exclusión}

Se incluyeron a los pacientes mayores de edad (18 años a más), posoperados de cirugía abdominal, a los cuales se les pudo realizar un tamizaje mediante el NRS 2002 y VGS para identificar su estado nutricional al ingreso y egreso. Por otro lado, se excluyeron a las mujeres gestantes o puérperas, pacientes referidos por complicaciones, posoperados en su lugar de origen y pacientes que no aceptaron formar parte del estudio.

\section{Instrumento de medición}

Para la recolección de las variables se preparó un instrumento, revisado por asesores en nutrición, cirugía y epidemiología. Se registraron como variables a la edad, sexo, diagnóstico operatorio y comorbilidades (obtenidas de la historia clínica, agrupadas según las 21 categorías de la clasificación internacional de enfermedades [CIE-10]), tipo de cirugía realizada (obtenida de la historia clínica y clasificada de acuerdo a los criterios del Manual de Clasificación y Codificación de Procedimientos Quirúrgicos y Anestesiológicos por grado de complejidad) [15]; presencia o ausencia del registro de peso y talla en el primer día de hospitalización (obtenida del cuaderno de ingresos del área de nutrición), día de inicio de dieta (obtenida de la historia clínica, según tres tipos, tolerancia a la vía oral, dieta líquida y dieta blanda) ${ }^{[16,17]}$; cumplimiento del consumo de dieta indicada (reportado por el paciente y/o del área de nutrición, clasificada en consume todo (>75\%), regular ( $25 \%-75 \%$ ) y nada ( $<25 \%)$, además características del consumo regular como anorexia, vómitos, náuseas, etc. ${ }^{[5]}$; reintervención quirúrgica, días de ayuno, uso de soporte nutricional, estancia hospitalaria y condición de egreso (obtenidas de la historia clínica). La variable principal fue la desnutrición durante la hospitalización, se definió en dos grupos: al grupo de pacientes que pasaron de bien nutrido (VGS A) a desnutrición moderada (VGS B), y a aquellos que pasaron de desnutrición moderada (VGS B) a desnutrición severa (VGSC) se les definió como «presente» y como «ausente» al resto de pacientes.

\section{Análisis estadístico}

Se analizaron los datos mediante el programa SPSS Statistics para Windows, versión 17.0 (SPSS Inc., Chicago, III., Estados Unidos). Para las variables categóricas, se realizó distribución de frecuencias y porcentajes. Para las variables cuantitativas, se utilizó media, desviación estándar y mediana. En el análisis bivariado, para variables cualitativas se aplicó la prueba exacta de Fisher. Asimismo, para el análisis entre las variables cuantitativas y cualitativas se aplicó prueba $U$ de Mann Whitney para las variables que sin distribución normal y $t$ de Student para las que tienen distribución normal. Para el análisis multivariado se aplicó regresión logística. Se usó un nivel de confianza al 95\% y un nivel de significancia de $p<0,05$.

\section{Aspectos éticos}

Se obtuvo la autorización del Comité de Investigación del Hospital Nacional Guillermo Almenara Irigoyen y el consentimiento firmado de los pacientes en estudio. Para proteger la confidencialidad de los datos obtenidos, se utilizó un código para la identificación del paciente.

\section{RESULTADOS}

Se evaluaron a 225 pacientes hospitalizados, de los cuales se excluyeron a $19(8,4 \%)$ porque fueron transferidos a un servicio clínico o unidad de cuidados intensivos, y otros no fueron intervenidos quirúrgicamente durante su estancia hospitalaria. De los 206 pacientes incluidos en el estudio, al realizar el tamizaje para la detección temprana de desnutrición con el NRS 2002 el $34,5 \%$ presentaron NRS $\geq 3$.

El 52,9\% eran hombres. Los pacientes de 60 años a más eran el $50,5 \%$, donde el $11,2 \%$ desarrolló desnutrición durante su estancia. Respecto al tamizaje y evaluación nutricional mediante NRS 2002 y VGS, al ingreso el 65,5\% estaban bien nutridos; el $30,1 \%$ estaban en riesgo de desnutrición moderada y el 4,4 \% en riesgo de desnutrición severa. La evolución nutricional de los pacientes durante la hospitalización el 5,8\% progresó de bien nutrido a desnutrición moderada al egreso; el 10,2 \% progresó de desnutrición moderada a desnutrición severa y el 16,1 \% 
mantuvo la condición de desnutrición moderada tanto al ingreso como egreso (Tabla 1).

En cuanto a las variables cuantitativas, el número de días de ayuno (media $\pm \mathrm{DE}: 7,70 \pm 3,88$ ), la estancia hospitalaria (media $\pm D E: 17,94 \pm 13,28$ ), y el día de inicio de la vía oral (media $\pm D E$ : $5,77 \pm 3,0$ ) mostraron asociación significativa (Tabla 2).

Tabla 2. Características de la desnutrición en pacientes hospitalizados en el servicio de cirugía de emergencia de un hospital del seguro social peruano

\begin{tabular}{|lcccc}
\hline \multicolumn{1}{|c}{ Variables } & Desnutrición & $\mathbf{n}$ & $\begin{array}{c}\text { Media } \pm \mathbf{D E} / \\
\text { Mediana (RIC) }\end{array}$ & $\begin{array}{c}\text { Valor } \\
\text { de } \mathbf{p}\end{array}$ \\
\hline \multirow{2}{*}{ Estancia } & Presente & 75 & $17,94 \pm 13,28$ & $<0,001^{*}$ \\
& Ausente & 131 & $9,43 \pm 13,28$ & \\
Días de ayuno & Presente & 75 & $7,70 \pm 3,88$ & $<0,001^{*}$ \\
& Ausente & 131 & $1,96 \pm 2,09$ & \\
Tolerancia oral & Presente & 13 & $5,77 \pm 3,03$ & $0,006^{*}$ \\
& Ausente & 44 & $3,34 \pm 2,63$ & \\
Dieta líquida & Presente & 17 & $7,88 \pm 5,89$ & $0,008^{*}$ \\
& Ausente & 133 & $3,50 \pm 3,62$ & \\
Dieta blanda & Presente & 20 & $9,05 \pm 5,26$ & $<0,001^{*}$ \\
& Ausente & 163 & $4,02 \pm 2,46$ & \\
Nutrición & Presente & 8 & $2(2)$ & $0,382^{\dagger}$ \\
enteral & Ausente & 14 & $3(2)$ & \\
Nutrición & Presente & 4 & $4(5,5)$ & $0,456^{\dagger}$ \\
parenteral & Ausente & 4 & $3(5,5)$ & \\
\hline
\end{tabular}

* T de Student

+ Prueba U de Mann-Whitney

DE: desviación estandar; RIC: rango intercuartila

El $49,5 \%$ presentaba comorbilidades a su ingreso, donde las enfermedades del sistema circulatorio fueron el $25,7 \%$. La mayoría de pacientes presentaban más de una comorbilidad a la vez. El 73,8 \% no tenía registro de peso y talla al ingreso, pero se observó que esta medición se realizó en el transcurso de la hospitalización en casi el 50 \% de pacientes. En cuanto a diagnósticos posoperatorios, el íleo paralítico y obstrucción intestinal sin hernia; y apendicitis aguda fueron los más frecuentes, representando el 25,2 \% cada uno. Los pacientes con obstrucción intestinal fueron los que mayormente agravaron su estado nutricional durante la hospitalización. El 8,3 \% tuvo reintervención quirúrgica, donde el 5,3\% deterioraron su estado nutricional basal. Respecto a la variación de ingesta de alimentos, el $27,7 \%$ tuvo un consumo regular. Entre las causas del consumo regular se encontró: anorexia $(13,1 \%)$ y náuseas $(8,3 \%)$ entre los más frecuentes. Solo el $12,4 \%$ de los posoperados recibió soporte nutricional. Se resalta que la mortalidad fue del $8,7 \%$, donde el 6,3\% habían agravado su estado nutricional (Tabla 3).

El análisis bivariado y multivariado se muestran en la Tabla 3. Según el análisis multivariado, se encontró asociación con desnutrición en pacientes hospitalizados en aquellos con alguna comorbilidad (OR: 2,32; IC 95\%:1,07-5,01; $p=0,03$ ); neoplasias (OR: 2,83; IC 95\%:1,21-6,63; $p=0,02$ ), enfermedades del sistema nervioso (OR: 5,66; IC 95\%: 1,24-25,82; $\mathrm{p}=0,05$ ); ausencia del registro de peso y talla (OR: 4,18; IC 95\%: 1,29-13,41; $p=0,01$ ); cirugía abdominal por íleo paralítico y obstrucción intestinal sin hernia (OR: 6,67; IC 95\%: 3,08-14,89; p<0,001); apendicitis aguda disminuía en $85 \%$ ( $p=0,004)$; colecistitis aguda disminuía en $91 \%$ ( $p=0,002$ ); Reintervención quirúrgica (OR: 13,91; IC 95\%: 4,8240,$06 ; p<0,001$ ); consumir en forma regular la dieta indicada (OR: 8,78; IC 95\%: 3,01-25,63; $p<0,001$ ); no consumir nada de la dieta indicada (OR: 39,97; IC 95\%: 11,39-140,23; $p<0,001$ ); el uso de soporte nutricional disminuía la exposición en un $83 \%(p<0,001)$. La desnutrición incrementaba 6 veces el riesgo de mortalidad. El número de días de ayuno, aumentaba $73 \%$ por cada día (OR: 1,73; IC 95\%: 1,31-2,27; p<0,001). (Tabla 3).

\section{DISCUSIÓN}

Al ingreso se halló que el $34,5 \%$ de pacientes estaba en riesgo nutricional, semejante a lo reportado en estudios internacionales ${ }^{[18,19]}$. Aunque menor a lo reportado por Correia et al. en Latinoamérica ${ }^{[20]}$. Estas diferencias pueden ser explicadas por el tipo de población estudiada, la falta de una definición universalmente aceptada de desnutrición, diferentes criterios diagnósticos empleados para definir el estado nutricional ${ }^{[21]}$, causas metodológicas y las características de cada hospital [22]. Sólo se consideró al servicio de Cirugía de Emergencia, por lo que las características nutricionales de los pacientes de este servicio pueden variar debido al estado pre y posoperatorio. El 5,8\% de los pacientes sin riesgo nutricional desarrollaron desnutrición moderada durante su estancia, cifra menor a la hallada por Álvarez et al. (9,6 \%) estudio en el que la población era pacientes con edad igual o mayor a 18 años y reclutados antes de las 48 horas posteriores al ingreso ${ }^{[18]}$. Además, el $16 \%$ de los pacientes presentó agravamiento de su estado nutricional durante su estancia hospitalaria. Este último dato, es superior a la encontrada en el ensayo clínico controlado realizado por Kondrup et al. en Dinamarca, donde hallaron que 14 de los 740 pacientes empeoraron su estado nutricional durante su hospitalización ${ }^{[13]}$. Esto demuestra que nuestros pacientes se encuentran a su ingreso, en situación de alto riesgo de desnutrición o padecen ya una desnutrición establecida. El estado nutricional influye en el bienestar del paciente y antes de iniciar cualquier terapia debe ser correctamente valorado ${ }^{[6]}$

En cuanto a la edad, no se apreció una asociación significativa con la desnutrición hospitalaria, discordante con otros estudios en España y en Latinoamérica ${ }^{[18,20]}$. La mayoría de los pacientes ancianos tienen problemas nutricionales, desde anorexia y pérdida de peso hasta caquexia ${ }^{[23]}$.

Se observaron que las neoplasias y enfermedades del sistema nervioso fueron las únicas que mostraron asociación significativa con la desnutrición hospitalaria, al igual que otros estudios realizados en España y Latinoamérica ${ }^{[18,20]}$. Este hallazgo corrobora lo que menciona la literatura, sobre desnutrición 
Tabla 3. Análisis bivariado y multivariado de factores asociados a desnutrición en pacientes hospitalizados en el servicio de cirugía de emergencia de un hospital del seguro social peruano

\begin{tabular}{|c|c|c|c|c|c|c|c|c|}
\hline \multirow{3}{*}{ Factores } & \multicolumn{4}{|c|}{ Desnutrición } & \multirow{2}{*}{\multicolumn{2}{|c|}{ Análisis bivariado }} & \multirow{2}{*}{\multicolumn{2}{|c|}{ Análisis multivariado † }} \\
\hline & \multicolumn{2}{|c|}{ Presente } & \multicolumn{2}{|c|}{ Ausente } & & & & \\
\hline & $\mathbf{n}$ & $\%$ & $n$ & $\%$ & OR (IC 95\%) & Valor de $\mathrm{p}^{*}$ & OR (IC 95\%) & Valor de $\mathbf{p}$ \\
\hline \multicolumn{9}{|l|}{ Sexo } \\
\hline Masculino & 21 & 10,2 & 88 & 42,7 & $1,34(0,38-4,72)$ & 0,640 & $1,69(0,79-3,60)$ & 0,180 \\
\hline Femenino & 12 & 5,8 & 85 & 41,3 & Referencia & & Referencia & \\
\hline \multicolumn{9}{|l|}{ Grupo etario } \\
\hline $18-40$ años & 7 & 3,4 & 52 & 25,2 & Referencia & 0,055 & Referencia & 0,053 \\
\hline 41-59 años & 3 & 1,5 & 40 & 19,4 & $0,58(0,16-2,14)$ & & $0,55(0,13-2,29)$ & \\
\hline$>60$ años & 23 & 11,2 & 81 & 39,3 & $1,86(0,85-4,08)$ & & $2,10(0,84-5,26)$ & \\
\hline Comorbilidad & 22 & 10,7 & 80 & 38,8 & $0,51(0,09-2,74)$ & 0,430 & $2,32(1,07-5,01)$ & 0,030 \\
\hline Enfermedades del sistema circulatorio & 11 & 5,3 & 42 & 20,4 & $0,68(0,11-3,95)$ & 0,660 & $1,55(0,70-3,44)$ & 0,280 \\
\hline Neoplasias & 10 & 4,9 & 23 & 11,2 & $0,55(0,11-2,71)$ & 0,460 & $2,83(1,21-6,63)$ & 0,020 \\
\hline $\begin{array}{l}\text { Enfermedades endocrinas, } \\
\text { nutricionales y metabólicas }\end{array}$ & 4 & 1,9 & 25 & 12,1 & $4,34(0,53-35,13)$ & 0,120 & $0,81(0,27-2,42)$ & 1,000 \\
\hline $\begin{array}{l}\text { Enfermedades del aparato } \\
\text { genitourinario }\end{array}$ & 5 & 2,4 & 10 & 4,9 & $0,32(0,03-3,42)$ & 0,350 & $2,91(0,96-8,81)$ & 0,070 \\
\hline Enfermedades del sistema nervioso & 3 & 1,5 & 3 & 1,5 & $0,51(0,02-9,43)$ & 0,650 & $5,66(1,24-25,82)$ & 0,050 \\
\hline Ausencia de registro de peso y talla* & 30 & 14,6 & 122 & 59,2 & $0,14(0,14-1,42)$ & 0,090 & $4,18(1,29-13,41)$ & 0,010 \\
\hline \multicolumn{9}{|l|}{ Diagnóstico postoperatorio } \\
\hline $\begin{array}{l}\text { Íleo paralítico y obstrucción } \\
\text { intestinal sin hernia }\end{array}$ & 20 & 9,7 & 32 & 15,5 & $6,46(0,86-48,56)$ & 0,070 & $6,77(3,08-14,89)$ & $<0,001$ \\
\hline Apendicitis aguda & 2 & 1,0 & 50 & 24,3 & $1,60(0,11-22,68)$ & 0,720 & $0,15(0,03-0,68)$ & 0,004 \\
\hline Colecistitis & 1 & 0,5 & 44 & 21,4 & $2,45(0,11-53,30)$ & 0,360 & $0,09(0,01-0,69)$ & 0,002 \\
\hline Colelitiasis & 1 & 0,5 & 13 & 6,3 & $4,31(0,18-101,39)$ & 0,3600 & $0,38(0,04-3,04)$ & 0,700 \\
\hline Peritonitis & 4 & 1,9 & 8 & 3,9 & $10,62(0,72-156,83)$ & 0,080 & $2,84(0,85-9,54)$ & 0,100 \\
\hline \multicolumn{9}{|l|}{ Tipo de cirugía realizada } \\
\hline $\begin{array}{l}\text { Cirugía mayor de mediana } \\
\text { complejidad (grado B) }\end{array}$ & 25 & 12,1 & 137 & 66,5 & $0,30(0,05-1,58)$ & 0,150 & $0,82(0,34-1,93)$ & 0,640 \\
\hline $\begin{array}{l}\text { Cirugía mayor de baja complejidad } \\
\text { (grado C) }\end{array}$ & 8 & 3,9 & 36 & 17,5 & Referencia & & Referencia & \\
\hline Reintervención quirúrgica & 11 & 5,3 & 6 & 2,9 & $0,82(0,12-5,63)$ & 0,840 & $13,91(4,82-40,06)$ & $<0,001$ \\
\hline \multicolumn{9}{|l|}{ Cumple consumo de dieta indicada } \\
\hline Todo & 5 & 2,4 & 123 & 59,7 & $0,07(0,01-0,70)$ & 0,020 & Referencia & $<0,001$ \\
\hline Regular & 15 & 7,3 & 42 & 20,4 & $0,19(0,02-1,32)$ & 0,090 & $8,78(3,01-25,63)$ & \\
\hline Nada & 13 & 6,3 & 8 & 3,9 & $3,00(0,53-16,74)$ & 0,210 & $39,97(11,39-140,23)$ & \\
\hline Uso de soporte nutricional & 11 & 5,3 & 14 & 10,7 & $3,00(0,53-16,74)$ & 0,210 & $0,17(0,07-0,42)$ & $<0,001$ \\
\hline \multicolumn{9}{|l|}{ Condición de egreso hospitalario } \\
\hline Fallecido ${ }^{\ddagger}$ & 13 & 6,3 & 5 & 2,4 & $21,8(7,27-65,14)$ & $<0,001$ & $6,78(4,10-11,23)$ & $<0,001$ \\
\hline Control por consultorio externo & 20 & 9,7 & 168 & 81,6 & Referencia & & Referencia & \\
\hline
\end{tabular}

Las categorías de comparación son contra ninguna comorbilidad o ningún diagnóstico.

OR: odds ratio, IC 95\%: intervalo de confianza al 95\%.

* En el primer día de hospitalización

† Se incluyeron las variables mostradas en la columna

‡ Riesgo relativo (el análisis ajustado incluyó las variables mostradas en la columna) 
en pacientes hospitalizados con cáncer, que varía entre el $30,9 \%$ y $60,9 \%{ }^{[24]}$. Esto se explica, debido a la ingesta baja de alimentos, absorción, incremento de los requerimientos nutricionales, mayor pérdida de nutrimentos, condicionado por las comorbilidades y la cirugía per se ${ }^{[23]}$.

El retraso del registro del peso y la talla, se asoció significativamente a la desnutrición durante la hospitalización. El hallazgo fue similar a lo reportado en la literatura, donde el $59,0 \%$ de los pacientes no es pesado ni tallado al ingreso ${ }^{[6]}$. El personal sanitario desconoce la importancia de una detección precoz, impidiendo el inicio de soporte nutricional ${ }^{[21]}$

La desnutrición puede favorecer la aparición de complicaciones como infección nosocomial, íleo prolongado y colección intraabdominal, causando reintervenciones quirúrgicas en más de una oportunidad y mayor morbimortalidad, como se observó en nuestro estudio y en el de Ecuador, este último incluyó 112 pacientes entre los 18 y 70 años de edad, ingresados durante un período de seis meses para cirugía programada o de emergencia ${ }^{[25]}$. Se debe resaltar, que las reintervenciones se relacionan, además, con la progresión de cada patología, hallazgos operatorios, condiciones generales y locales del paciente, y en algunas ocasiones iatrogenia ${ }^{[26]}$.

El número de días de ayuno en pacientes desnutridos es inadecuado según la Sociedad Americana de Nutrición Parenteral y Enteral (ASPEN), quien establece inadecuado un ayuno mayor a siete días en bien nutridos y mayor a cinco días en desnutridos ${ }^{[27]}$. Lo cual empeora los resultados clínicos de estos pacientes de manera independiente de otras condiciones como la edad o enfermedad, observado en este estudio y en España ${ }^{[28]}$. En cuanto al día de inicio de la vía oral, los resultados mostrados en la Tabla 2 , contrastan con los de México, en un estudio con 88 pacientes sometidos a cirugía abdominal mayor, la mediana de tiempo de inicio de vía oral fue de 18 horas desde el término de la cirugía, mientras que la de dieta blanda fue de 42 . El número de días de ayuno y el inicio de la vía oral encontrado, se explica debido a que, muchas veces está sujeto al cese del íleo posoperatorio, que es una consecuencia adversa inevitable de los procedimientos quirúrgicos abdominales. Lo habitual ha sido retrasar el inicio de la nutrición oral hasta asegurarse de la presencia de ruidos intestinales, flatos y/o evacuaciones; pero cuando se revisa la evidencia publicada, han demostrado que la presencia de estos no tiene valor en la indicación de la dieta ${ }^{[16]}$. Cabe mencionar que, el $62,1 \%$ consumió más del $75 \%$ de la dieta indicada, similar a lo reportado en un estudio de México ${ }^{[5]}$. En referencia al desecho alimentario, las razones principales son debidas a factores clínicos, alimentarios y dietéticos, de servicio y ambientales. A mayor desperdicio alimentario, menor ingesta de alimentos y, por tanto, mayor pérdida de peso de las personas hospitalizadas ${ }^{[19]}$.

El 14,6\% recibió soporte nutricional, lo que representa el 36,4\% de pacientes desnutridos al alta y el $34,5 \%$ de pacientes con desnutrición al ingreso, similar a otros estudios ${ }^{[18]}$. Otra de las variables de nuestro estudio, que también tuvo asociación, fue el tiempo de hospitalización. La estancia hospitalaria fue mayor en los pacientes desnutridos, como era de esperar por la asociación de desnutrición y complicaciones ${ }^{[29]}$. En España,
Álvarez et al. ${ }^{[18]}$ identificaron que el tiempo de hospitalización en desnutridos era 15 vs. 8 días en no desnutridos $(p<0,001)$. Las comorbilidades, reintervenciones quirúrgicas y la disminución de la ingesta de alimentos, con algunos factores que generan un mayor tiempo de hospitalización ${ }^{[30]}$. La mayoría de los pacientes eran clasificados como nivel de atención terciaria, es decir, son admitidos cuando los síntomas ya están avanzados, donde la enfermedad subyacente requiere también atención nutricional. En cuanto a la mortalidad, el hallazgo fue similar a la del Hospital Nacional Hipólito Unanue (Perú), en el que se evaluó a 136 pacientes mayores de 15 años en quienes se realizó algún tipo de sutura gastrointestinal o anastomosis ${ }^{[31]}$. Esto se explica, ya que la cirugía per se condiciona ayuno de tiempo variable, estrés quirúrgico y aumento de la tasa metabólica. Estos problemas son mayores si la cirugía es gastrointestinal, llevando a la consunción de las reservas y de no tratarse, a alteraciones estructurales que pueden llevar a la muerte como se ha observado ${ }^{[31,32]}$.

El haber encontrado la apendicitis aguda y colecistitis aguda como factores que disminuían la desnutrición hospitalaria, podría estar explicada por el tiempo de estancia de estas patologías, que es mucho menor que las demás causas de cirugía; además que se presentan en edades más tempranas donde la desnutrición es menos probable que se presente ${ }^{[20]}$.

Referente a las limitaciones, no se utilizó variables bioquímicas para evaluar desnutrición, ya que esta demanda un costo económico superior y muchas veces no está al alcance de los hospitales para realizar el tamizaje nutricional, a diferencia de los test utilizados. Sobre el consumo total o parcial de la dieta indicada, se consignaron respuestas verbales, más no mediciones reales, lo cual generaría un sesgo de medición.

En conclusión, los factores asociados a desnutrición durante la hospitalización fueron: presentar alguna comorbilidad, neoplasias y enfermedades del sistema nervioso; ausencia del registro de peso y talla, cirugía abdominal por íleo paralítico y obstrucción intestinal sin hernia, reintervención quirúrgica, número de días de ayuno, día de inicio de la vía oral, consumir en forma regular o nada de la dieta indicada, y el número de días de ayuno. Como factores protectores de desnutrición hospitalaria se presentaron a la apendicitis aguda, colecistitis aguda y el uso de soporte nutricional.

Es importante realizar un tamizaje para la detección temprana de desnutrición al ingreso de los pacientes que serán hospitalizados y posteriormente una evaluación nutricional en aquellos que estén en riesgo.

Contribuciones de autoría: todos los autores participaron en la redacción o revisión crítica de este manuscrito para contenido intelectual importante, y todos los autores aprobaron la versión final que se publicará. PAZH y CDV tienen acceso total a todos los datos del estudio y se responsabilizan por su integridad y la precisión de los análisis realizados.

\section{Potencial conflictos de intereses: ninguno.}

Fuente de financiamiento: autofinanciado. 
ORCID:

Paola Alejandra Zeña-Huancas: https://orcid.org/0000-0002-6279-8316 Denissa Pajuelo-García: https://orcid.org/0000-0003-1158-9132 Cristian Díaz-Vélez: https://orcid.org/0000-0003-4593-2509

\section{REFERENCIAS BIBLIOGRÁFICAS}

1. Giryes S, Leibovitz E, Matas Z, Fridman S, Gavish D, Shalev B, et al. Measuring Nutrition Risk in Hospitalized Patients: MENU, A HospitalBased Prevalence Survey. Isr Med Assoc J. 2012;14(7):405-409.

2. Universidad Nacional Mayor de San Marcos. Cirugía : I cirugía general. Lima: UNMSM; 1999.

3. Schiesser M, Muller S, Kirchhoff P, Breitenstein S, Schafer M, Clavien P. Assessment of a novel screening score for nutritional risk in predicting complications in gastro-intestinal surgery. Clin Nutr. 2008;27(4):565570. doi: 10.1016/j.clnu.2008.01.010.

4. Gómez M, González F, Sánchez C. Estudio del estado nutricional en la población anciana hospitalizada. Nutr Hosp. 2005;20(4):286-92.

5. Fuchs V, Mostkoff D, Gutiérrez G, Amancio O. Estado nutricio en pacientes internados en un hospital público de la ciudad de México. Nutr Hosp. 2008;23(3):294-303.

6. Santana S. Comentario al artículo: El esqueleto en la taquilla del hospital. Nutr. Hosp. 2005;20(4):297-307.

7. Norman K, Pichard C, Lochs H, Pirlich M. Prognostic impact of diseaserelated malnutrition. Clin Nutr 2008;27(1):5-15. doi: 10.1016/j. clnu.2007.10.007.

8. García A, Álvarez J, Planas M, Burgos, Araujo K. Multidisciplinary consensus on the approach to hospital malnutrition in Spain. Nutr Hosp. 2011; 26(4):701-710. doi: 10.1590/S0212-16112011000400006.

9. Committee of Experts on Nutrition, Food and Consumer Health (Partial Agreement) (P-SC-NU). Nutrition in care homes and home care. Report and recommendations: from recommendations to action [Internet]. Council of Europe Publishing; 2009 [citado el 12 de febrero de 2020]. Disponible en: https://dske.dk/Politik/Internationalt/rapport_fra_sub_ commitee_under_europaraadet_dec_08.doc.pdf.

10. Canicoba M. Documento de consenso: funciones y competencias del nutricionista clínico: Revisión de diferentes posiciones de sociedades científicas latinoamericanas [Internet]. Federación Latinoamericana de Terapia Nutricional y Nutrición Clínica y Metabolismo (FELANPE): 2012 [citado el 17 de febrero de 2020]. Disponible en: http:// felanpeweb.org/wp-content/uploads/2015/11/Consenso-15-deNoviembre-2012.pdf.

11. Velasco C, García E, Rodríguez V, Frias L, Garriga R, Álvarez J, et al. Comparison of four Nutritional Screening Tools to Detect Nutritional Risk in Hospitalized Patients. A multicenter study. Eur J Clin Nutr. 2011;65(2):269-74. doi: 10.1038/ejcn.2010.243.

12. Valero M, Díez L, El Kadaoui N, Jiménez A, Rodríguez H, León M. ¿Son las herramientas recomendadas por la ASPEN y la ESPEN equiparables en la valoración del estado nutricional?. Nutr Hosp. 2005;20(4):259-267.

13. Kondrup J, Rasmussen HH, Hamberg O, Stanga Z, ESPEN Working Group. Nutritional Risk Screening (NRS 2002): a new method based on an analysis of controlled clinical trials. Clin Nutr. 2003;22(3):321-36. doi: 10.1016/s0261-5614(02)00214-5.

14. Destky AS, McLaughlin JR, Baker JP, Johnston N, Whittaker S, Mendelson RA, et al. What is Subjective Global Assessment of Nutritional Status?. J Parenter Enteral Nutr. 1987;11(1):8-13. doi: 10.1177/014860718701100108.

15. Conareme. Estándares mínimos de formación para el programa de segunda especialización en Cirugía General [Internet]. Lima: Conareme; 2001 [citado el 20 de febrero de 2020]. Disponible en: http://www. conareme.org.pe/web/Documentos/Estandares\%20Minimos/ GRUPO\%20II/cirugia\%20oncologica.pdf.
16. Hernández-Hernández B, Figueroa-Gallaga L, Sánchez-Castrillo C, Belmonte-Montes C. Utilidad de la presencia de ruidos intestinales, flatos y evacuación en la predicción de la tolerancia a la vía oral en pacientes sometidos a cirugía abdominal mayor. Rev Gastroenterol Mex. 2007;72(3):207-213.

17. Mesejo A, Martínez J, Martínez C. Manual básico de nutrición clínica y dietética [Internet]. Valencia: Hospital Clínico Universitario de Valencia; 2012 [citado el 22 de febrero de 2020]. Disponible en: https://docplayer.es/6728073-Manual-basico-de-nutricion-clinicay-dietetica.html.

18. Álvarez J, Planas $\mathrm{M}$, León $\mathrm{M}$, García $\mathrm{A}$, Celaya $\mathrm{S}$, García $\mathrm{P}$, et al. Prevalence and costs of malnutrition in hospitalized patients; the PREDyCES Study. Nutr Hosp. 2012;27(4):1049-1059.

19. Monferrer S, Fernández M, Murillo J. Desnutrición y factores que influyen en la ingesta de alimentos en pacientes hospitalizados: una revisión. Nutr clín diet hosp. 2014;34(3):80-91. doi: 10.12873/343salvadormonferrer.

20. Correia I, Campos A. Prevalencia de la Malnutrición Hospitalaria en Latinoamérica: El Estudio Multicentrico ELAN. Nutrition. 2003;19:823825.

21. Ocón M, Altemir J, Mañas A, Sallán L, Aguillo E, Gimeno J. Comparación de dos herramientas de cribado nutricional para predecir la aparición de complicaciones en pacientes hospitalizados. Nutr Hosp. 2012;27(3):701-706. doi: 10.3305/nh.2012.27.3.5724.

22. Vidal A, Iglesias M, Pertega S, Ayúcar A, Vidal O. Prevalencia de malnutrición en los servicios médicos y quirúrgicos de un hospital universitario. Nutr Hosp. 2008;23(3):263-267.

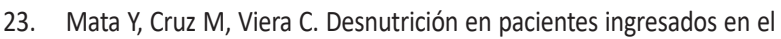
servicio de medicina, Hospital Universitario Dr. Luis Razetti, Barcelona, Estado Anzoátegui, julio-diciembre del 2009 [Tesis maestría]. Barcelona, España; 2010.

24. Veramendi L, Zafra J, Salazar O, Basilio J, Millones E, Pérez G, et al. Prevalencia y factores asociados a desnutrición hospitalaria en un hospital general; Perú, 2012. Nutr Hosp. 2013;28(4):1236-1243. doi: 10.3305/nh.2013.28.4.6390

25. Estrada F, Salazar S. Riesgo de complicaciones postoperatorias en pacientes desnutridos sometidos a cirugía. Rev Fac Cien Med Quito. 2006;31(3-4):74-76.

26. Ortiz, Pompa C, Cruz R. Riesgo de reintervención quirúrgica abdominal; Estudio de casos y controles. Revista de Especialidades MédicoQuirúrgicas. 2005;10(2):25-28.

27. White J, Guenter P, Jensen G, Malone A, Schofield M. Consensus statement: Academy of Nutrition and Dietetics and American Society for Parenteral and Enteral Nutrition: characteristics recommended for the identification and documentation of adult malnutrition (undernutrition). J Parenter Enteral Nutr. 2012;36(3):275-83. doi: 10.1177/0148607112440285.

28. Kyriakos G, Calleja A, Ávila D, Cano I, Ballesteros M, Vidal A. Prolonged fasting with fluid therapy is related to poorer outcomes in medical patients. Nutr Hosp. 2013;28(5):1710-1716. doi: 10.3305/ nh.2013.28.5.6693.

29. Kyle U, Coss-Blu J. Nutritional assessment and length of hospital stay. CMAJ. 2010;108(17):1831-2. doi: 10.1503/cmaj.101256.

30. Ordoñez A, Madalozzo M, Cestonaro T, Cardoso J, Ligocki A. Nutritional status influences the length of stay and clinical outcomes in hospitalized patients in internal medicine wards. Nutr Hosp. 2013;28(4):1313-1320. doi: $10.3305 /$ nh.2013.28.4.6609.

31. Alvarez D, Revoredo F, Suarez M, Acevedo I, Lloclla P. Estado Nutricional y Morbi-Mortalidad en Pacientes con Anastomosis Gastrointestinales en el Hospital Nacional Hipolito Unanue (HNHU). Rev Gastroenterol Perú. 2012;32(3):273-280.

32. Lozano-Ballena S, Meléndez-Ramírez F, Poma-Ortiz J, Díaz-Vélez C. Estado nutricional del paciente adulto mayor hospitalizado usuario de sonda nasogástrica en un Hospital del Seguro Social, Perú. Revista Del Cuerpo Médico Del HNAAA. 2019;11(3):142-148. 